\title{
INDEX OF DIRAC OPERATOR AND SCALAR CURVATURE ALMOST NON-NEGATIVE MANIFOLDS*
}

\author{
FUQUAN FANG ${ }^{\dagger}$
}

\begin{abstract}
A manifold $M$ is called scalar curvature almost non-negative if for any constant $\varepsilon>0$, there is a Riemannian metric $g$ on $M$ such that $s_{g} \cdot \operatorname{diam}(M, g)^{2} \geq-\varepsilon$ and the sectional curvature $\operatorname{Sec}_{g} \leq 1$, where $s_{g}$ (resp. $\operatorname{diam}(M, g)$ ) is the scalar curvature (resp. diameter) of $(M, g)$.

Among others we prove that for a scalar curvature almost non-negative manifold $M$ with $\hat{A}(M) \neq 0$ (resp. $\mathcal{A}(M) \in \mathbb{Z}_{2}$ nonzero and $\chi(M) \neq 0$ if $\left.n=2(\bmod 8)\right)$, there is a constant $\varepsilon(n)>0$ such that, if the scalar curvature $s_{M} \geq-\varepsilon(n)$, then

(i) the fundamental group $\pi_{1}(M)$ is finite;

(ii) $M$ admits a real analytic Ricci flat metric $g_{0}$ such that its Riemannian universal covering $\tilde{M}$ is isometric to the product of Ricci flat Kähler-Einstein manifolds and/or Joyce manifolds of dimension 8 with special holonomy group $\operatorname{Spin}(7)$.
\end{abstract}

0. Introduction. Let $M$ be a closed Spin manifold. It is well-known that $M$ admits a metric with positive scalar curvature only if the index of the Dirac operator vanishes, by the classical Lichnerowicz formula and the Atiyah-Singer index theorem (cf. [LM]). The Gromov-Lawson conjecture, confirmed by Stolz [St], asserts the converse for simply connected Spin manifolds of dimension at least 5 . There are many manifolds with non-negative scalar curvature but do not accept metrics of positive scalar curvature, e.g. torus, $K_{3}$-surfaces, etc.

A basic theorem of Bourguignon shows that such a Spin manifold must be Ricci flat. Starting from [St], Futaki [Fu] characterized all simply connected Spin manifolds of dimension at least 5 with non-negative scalar curvature. It turns out that such a manifold either admits a metric with positive scalar curvature, or it is the product of Ricci flat Kähler-Einstein manifolds and/or Joyce manifold of dimension 8 with Spin(7)-holonomy (manifolds in the latter class are called rigidly scalar flat). A keypoint involved is that, every harmonic spinor must be parallel and therefore, the holonomy group must be special (cf. [Fu] [Wa]).

Recall that a manifold is called almost flat (resp. almost Ricci flat) if for any positive constant $\varepsilon>0$, there is a Riemannian metric $g$ on $M$ such that

$$
\left|\operatorname{Sec}_{g} \cdot \operatorname{diam}(M, g)^{2}\right| \leq \varepsilon\left(\operatorname{resp}\left|\operatorname{Ric}_{g} \cdot \operatorname{diam}(M, g)^{2}\right| \leq \varepsilon\right)
$$

where $\operatorname{Sec}_{g}$ (resp. $\mathrm{Ric}_{g}$ ) is the sectional (resp. Ricci) curvature.

The celebrated Gromov theorem asserts that an almost flat manifold must be an infra-nilmanifold, i.e. a finite regular cover must be a nilmanifold. By the Bochner technique it is also well-known that an almost Ricci flat manifold has the first Betti number at most $n$, where $n$ is the dimension. In contrast, however, very recently Lohkamp [Lo] proved that for every compact manifold $M$ of dimension at least 3 and for any given positive constant $\varepsilon$, there is a metric $g$ so that its scalar curvature satisfies that $\left|s_{g} \cdot \operatorname{diam}(M, g)^{2}\right| \leq \varepsilon$.

We call a manifold $M$ is of scalar curvature almost non-negative if for any constant $\varepsilon>0$, there is a Riemannian metric $g$ on $M$ such that $s_{g} \cdot \operatorname{diam}(M, g)^{2} \geq-\varepsilon$ and the sectional curvature $\operatorname{Sec}_{g} \leq 1$, where $s_{g}(\operatorname{resp} . \operatorname{diam}(M, g))$ is the scalar curvature

* Received July 13, 2002; accepted for publication December 13, 2002.

$\dagger$ Nankai Institute of Mathematics, Nankai University, Tianjin 300071, P.R.C. (ffang@nankai.edu.cn). Supported by NSFC Grant 19741002, RFDP, CNPq and the Qiu-Shi Foundation. 
(resp. diameter) of $(M, g)$. Note that almost flat manifolds must be of scalar curvature non-negative.

The main purpose of the present paper is to characterize manifolds with almost non-negative scalar curvature. We will prove that there are indeed nontrivial topological obstructions for manifolds being of scalar curvature almost non-negative. For example, following from our theorem the connected sum, $K_{3} \# S^{1} \times S^{3}$, of a $K_{3}$-surface with $S^{1} \times S^{3}$, does not admits metrics of almost non-negative scalar curvature.

Let $M$ be Spin manifold. Let $S^{+}$(resp. $S^{-}$) be the Spinor bundles on $M$. Let $\mathcal{D}^{+}: \Gamma\left(S^{+}\right) \rightarrow \Gamma\left(S^{-}\right)$denote the.Dirac operator. The Atiyah-Singer index theorem asserts that ind $\left(\mathcal{D}^{+}\right)=\hat{\mathcal{A}}(M)$ is a Spin cobordism invaraint and defines a ring homomorphism

$$
\mathcal{A}: \Omega_{*}^{S p i n} \rightarrow K O^{-*}(p t)
$$

which is non-zero only if the dimension is $0(\bmod 4)$ or $1,2(\bmod 8)$. In particular, if $n=0(\bmod 4), \mathcal{A}(M)=\hat{A}(M)$ is the $\hat{A}$-genus of $M$.

Let $\mathcal{M}(n, d)$ denote the set of all closed Riemannian $n$-manifolds such that the sectional $K_{M} \leq 1$, and $\operatorname{diam} M \leq d$.

A manifold $M \in \mathcal{M}(n, d)$ is called non-collapsing if there is no a sequence of metrics of bounded curvature collapsing to a lower dimensional space in the GromovHausdorff topology.

Theorem A. Let $M \in \mathcal{M}(n, d)$ be a closed non-collapsing Spin manifold such that $\mathcal{A}(M) \neq 0$. There is a constant $\varepsilon(n)>0$ such that if the scalar curvature $s_{M} \geq-\varepsilon(n)$, then $M$ admits a Ricci flat real analytic Riemannian metric $g_{0}$ with restricted holonomy group $\operatorname{Hol}^{0}\left(M, g_{0}\right)$ a product whose irreducible components are $S U(m), S p(m), G_{2}$ or $\operatorname{Spin}(7)$.

By the local formula for $\hat{A}$-genus (Euler characteristic), $M \in \mathcal{M}(n, d)$ is noncollapsing if $\hat{A}(M) \neq 0$ (resp. the Euler characteristic $\chi(M) \neq 0$ ). Therefore Theorem A together with the Cheeger-Gromoll splitting theorem [CGr] implies immediately

Corollary 0.1. Let $M \in \mathcal{M}(n, d)$ be a closed Spin manifold. Suppose that $\hat{A}(M) \neq 0$ (resp. $\mathcal{A}(M) \neq 0$ and $\chi(M) \neq 0$ if $n=2(\bmod 8))$. There is a constant $\varepsilon(n)>0$ such that, if the scalar curvature $s_{M} \geq-\varepsilon(n)$; then

(i) the fundamental group $\pi_{1}(M)$ is finite;

(ii) $M$ admits a real analytic Ricci flat metric $g_{0}$ such that its Riemannian universal covering $\tilde{M}$ is isometric to the product of Ricci flat Kähler-Einstein manifolds and/or Joyce manifolds of dimension 8 with special holonomy group Spin(7).

The above theorem together with the Stolz theorem [St] clearly implies

Corollary 0.2. Let $M \in \mathcal{M}(n, d)$ be a closed non-collapsing Spin manifold. If $M$ is simply connected and $n \geq 5$. There is a constant $\varepsilon(n)>0$ such that, if the scalar curvature $s_{M} \geq-\varepsilon$, then either $M$ admits a metric of positive scalar curvature or admits a Ricci flat metric.

The proof of the Theorem A together with Corollaries 3.1 and 3.2 in [Wa] imply the following two corollaries

Corollary 0.3. Let $M \in \mathcal{M}(4, D)$ be a closed Spin manifold so that $\hat{A}(M) \neq 0$. There is a constant $\varepsilon>0$ such that, if the scalar curvature $s_{M} \geq-\varepsilon$, then $M$ is bi- 
Lipschitz isometric to a Calabi-Yau $K_{3}$-surface.

Corollary 0.4. Let $M \in \mathcal{M}(8, D)$ be a closed Spin manifold. Assume that $\hat{A}(M) \neq 0$. There is a constant $\varepsilon>0$ such that, if the scalar curvature $s_{M} \geq-\varepsilon$, then $M$ is simply connected.

Observe that the $\hat{A}$-genus of a $K_{3}$-surface $\hat{A}\left(K_{3}\right) \neq 0$. Corollary 0.1 implies easily that the connected sum $K_{3} \# S^{1} \times S^{3}$ does not admit an almost non-negative scalar curvature metric. This also answers in negative the following question:

Problem 0.5. Let $M$ (resp. $N$ ) be a manifold with positive (resp. non-negative) scalar curvature. Does the connected sum $M \# N$ admit a metric with almost nonnegative scalar curvature?

This is maybe interesting by comparing with the amazing discovery of SchoenYau [SY], that the connected sum of any two $n$-manifolds $(n \geq 3)$ with positive scalar curvature admits a metric with positive scalar curvature.

Some remarks on the above results are in order.

REMARK 0.6. The Seiberg-Witten theory [Wi] implies that 4-manifolds with non-trivial Seiberg-Witten invariant do not accept any metric with positive scalar curvature. Furthermore, Witten ([Wi]) proved that a scalar curvature non-negative 4manifold with non-trivial Seiberg-Witten invariant is hyper-Kähler, if $b_{2}^{+} \geq 2$. SeibergWitten theory may be used to study scalar curvature almost non-negative 4-manifolds in the spirit of the presented paper.

REMARK 0.7. Theorem A should be compared with a recent result in [Lot] where sectional curvature is assumed being almost non-negative.

REMARK 0.8. Theorem A holds identically, if the upper bound for the sectional curvature is replaced by a upper bound for the Ricci curvature together with a positive lower bound for the conjugate radii.

Now let us start to sketch the idea in the proof of Theorem A.

Suppose Theorem A is false, then there is a sequence of $n$-dimensional Spin manifolds $\left(M_{i}, g_{i}\right)$ so that $\mathcal{A}\left(M_{i}\right) \neq 0$, the scalar curvatures $s_{g_{i}} \geq-1 / i$, the diameter diam $M_{i} \leq D$ and $\operatorname{Sec}_{g_{i}} \leq 1$ for some positive constant $D$. By the Gromov precompactness theorem and the Cheeger-Gromov theorem, passing to a subsequence if necessary, we may assume that $\left(M_{i}, g_{i}\right)$ converges in $L^{2, p}$-class to a manifold $\left(X, g_{\infty}\right)$ (since $M_{i}$ are non-collapsing.) Let $\phi_{i} \in \Gamma\left(S_{i}^{+}\right)$be a harmonic spinor with unit $L^{2}$-norm. Using the Lichnerowicz formula and the scalar curvature bound we prove that $\phi_{i}$ converges to a parallel harmonic spinor $\phi_{\infty}$ with nontrivial norm. Therefore $\phi_{i}$ is almost parallel for $i$ large, which implies that the Ricci curvature of $\left(M_{i}, g_{i}\right)$ converges in $L^{p}$-class to zero. By regularity of Einstein equation we know that there is a real analytic Ricci flat metric on $X$. A contradiction.

1. Preliminaries. In this section we give some necessary preliminary results needed in next sections.

\section{a). Harmonic coordiantes}

A local coordinate $\left(h^{1}, \ldots, h^{n}\right)$ is harmonic if each component is a harmonic function, i.e., $\Delta h^{i}=0$ for $i=1, \ldots, n$, where $\Delta$ is the Laplacian opertator. In a harmonic 
coordinate, the Ricci curvature of the metric tensor $g$ satisfies the equation

$$
\left(\mathrm{Ric}_{g}\right)_{i j}=-\frac{1}{2} \triangle g_{i j}+Q(g, \partial g)
$$

here $g_{i j}=g\left(\frac{\partial}{\partial h^{2}}, \frac{\partial}{\partial h^{j}}\right)$ and $Q(.,$.$) is a quadratic form of its variables (c.f. [Pe]).$

The existence of harmonic coordinate ball of uniform radius is studied in [JK] [An], in terms of various curvature bound. We quote the following

ThEOREM 1.1 [AN]. Let $(M, g)$ be a Riemannian n-manifold (not necessarily complete) such that

$$
\left|R i c_{M}\right| \leq \lambda, \operatorname{inj} B\left(x, \frac{1}{2} \cdot \operatorname{dist}(x, \partial M)\right) \geq i_{0}(x)>0
$$

Then, given any $C>1$ and $\alpha \in(0,1)$, there is an $\varepsilon_{0}=\varepsilon_{0}(\lambda, C, n, \alpha)$ with the following property: given any $x \in M$, there is a harmonic coordinate system $U=\left\{u_{i}\right\}_{1}^{m}$ defined on $B(x, \varepsilon(x)) \subset M$ such that if $g_{i j}=g\left(\nabla u_{i}, \nabla u_{j}\right)$, then $g_{i j}(x)=\delta_{i j}$ and

$$
C^{-1} \cdot I \leq g(y) \leq C \cdot I, \text { (as bilinear forms) }
$$

for all $y \in B(x, \varepsilon(x))$ (resp. $\left.\frac{\varepsilon(x)^{2-\frac{n}{p}}}{\sqrt{\operatorname{vol} B(x, \varepsilon(x))}}\left\|g_{i j}\right\|_{L^{2, p}(B(x, \varepsilon(x))} \leq C\right)$, where

$$
\frac{\varepsilon(x)}{i_{0}(x)} \geq \varepsilon_{0} \cdot \frac{\operatorname{dist}(x, \partial M)}{\operatorname{diamM}}>0
$$

where $B(x, r)$ is the metric ball with radius $r, I$ the identity matrix and inj is the injectivity radius.

\section{b). Compactness theorems}

THEOREM 1.2 [CG]. Let $\left(M_{i}, g_{i}\right)$ be a sequence of compact Riemannian manifolds whose sectional curvature, diameter, and injectivity radius satisfy

$$
\lambda \leq S e c \cdot g_{i} \leq \Lambda, \text { diam } \leq d, \quad i_{M}>i_{0},
$$

where the constants are independent of $i$. Then, replacing $M_{i}$ by a subsequence if necessary, $M_{i}$ converges to a metric space $X$, such that

(i) $X$ is a differentiable manifold;

(ii) there is a diffeomorphsim $f_{i}: X \rightarrow M_{i}$ for all sufficiently large $i$;

(iii) the pullback metrics $f_{i}^{*}\left(g_{i}\right)$ converges in $C^{1, \alpha}$-class to a $C^{1, \alpha}\left(\right.$ resp. $\left.L^{2, p}\right)$ Riemannian metric $g_{\infty}$ in $X$, for any prescribed real number $\alpha \in(0,1)$ (resp. positive integer $p>1$ ).

For the sake of simplicity, in the rest of the paper we fix the real number $\alpha \in(0,1)$ (resp. the integer $p>n$ ).

For a sequence $\left(M_{i}, g_{i}\right)$ as above, the limit metric $g_{\infty}$ is not necessarily $C^{2}$. However, if the $L^{p}$-Ricci curvature (for $p$ large) of $g_{\infty}$ vanishes identically, then by the elliptic regularity of the Einstein equation one may conclude the smoothness of $g_{\infty}$ (indeed real analytic). That is 
THEOREM $1.3[\mathrm{AN}]$. Let $\left(M_{i}, g_{i}\right)$ be a sequence as in Theorem 1.2. If the $L^{p}$ Ricci curvature (for some large $p$ ) of $g_{\infty}$ vanishes identically. Then $\left(M_{i}, g_{i}\right)$ has a subsequence converging to a $C^{\infty}$ Riemannian manifold $X$, such that

(i) there is a diffeomorphsim $f_{i}: X \rightarrow M_{i}$ for all sufficiently large $i$;

(ii) the pullback metrics $f_{i}^{*}\left(g_{i}\right)$ converges in $C^{\infty}$-class to a real analytic Riemannian metric $g_{\infty}$.

2. Convergence of harmonic spinors. Let $M$ be a closed Spin Riemannian manifold. Let $S^{+}$(resp. $S^{-}$) be the positive (resp. negative) spinor bundle. Let $D: \Gamma\left(S^{+}\right) \rightarrow \Gamma\left(S^{-}\right)$be the Dirac operator. The classical Lichnerowicz formula reads

$$
D^{2}=\nabla^{*} \nabla+\frac{1}{4} s
$$

where $s$ is the scalar curvature of $M$.

Let $M_{i}$ be a sequence of closed Spin Riemannian $n$-manifolds such that

$$
\operatorname{diam}\left(M_{i}\right)^{2} \cdot s_{i} \geq-\varepsilon_{i} ; \operatorname{Sec}_{g_{i}} \leq 1
$$

where $s_{g_{i}}$ is the scalar curvature of $M_{i}$. By rescaling we may assume that $\operatorname{diam}\left(M_{i}\right) \leq$ 1 and $s_{g_{i}} \geq-\varepsilon_{i}$. Note that the sectional curvature $\operatorname{Sec}_{g_{i}} \geq-\lambda(n)$.

Let $\phi_{i} \in \Gamma\left(S_{i}^{+}\right)$denote the harmonic spinors (i.e. $D_{i} \phi_{i}=0$.) By normalizing we may assume that

$$
\frac{1}{\operatorname{vol}\left(M_{i}\right)} \int_{M_{i}}\left|\phi_{i}\right|^{4}=1
$$

Lemma 2.3. Let $\phi_{i}$ be as above. Then

$$
\left|\phi_{i}\right|_{L^{\infty}} \leq C
$$

where $C$ is a constant depending only on $n, \varepsilon_{i}$ and $\lambda(n)$.

Proof. Note that

$$
\Delta\left|\phi_{i}\right|^{2}=2\left\langle\nabla_{i}^{*} \nabla_{i} \phi_{i}, \phi_{i}\right\rangle-2\left|\nabla_{i} \phi_{i}\right|^{2}
$$

By the Lichnerowicz formula we get that

$$
0=\nabla_{i}^{*} \nabla_{i} \phi_{i}+\frac{1}{4} s_{g_{i}} \phi_{i}
$$

Therefore

$$
\Delta\left|\phi_{i}\right|^{2} \leq-\frac{1}{2} s_{g_{i}}\left|\phi_{i}\right|^{2} \leq \frac{1}{2} \varepsilon_{i}\left|\phi_{i}\right|^{2}
$$

By [Ga] Proposition 3.2 it follows that

$$
\left|\phi_{i}\right|_{L^{\infty}}^{2} \leq C_{1} \frac{\left(\int_{M_{i}}\left|\phi_{i}\right|^{4}\right)^{\frac{1}{2}}}{\sqrt{\operatorname{vol}\left(M_{i}\right)}}=C_{1}
$$

The desired result follows.

Let $2 r$ denote a uniform lower bound for the conjugate radii of $M_{i}$. By Theorem 1.1 , there are radii $r$ balls, $B_{i}^{1}(r), \cdots, B_{i}^{m}(r)$, in the tangent spaces $T_{p_{k}^{i}} M_{i}$, where $p_{1}^{i}, \cdots, p_{m}^{i} \in M_{i}$ are $m$ points so that the exponential maps $\exp _{p_{k}^{i}}: B_{i}^{k}(r) \subset T_{p_{k}^{i}} M_{i} \rightarrow$ $M_{i}$ are embeddings and $\exp _{p_{1}^{i}}\left(B_{i}^{1}(r)\right), \cdots, \exp _{p_{m}^{i}}\left(B_{i}^{m}(r)\right)$ is a harmonic coordinate 
covering of $M_{i}$. Fixing $k$, consider the pullback metric $\exp _{p_{k}^{i}}^{*}\left(g_{i}\right)$ on $B_{i}^{k}(r)$. By Theorem 1.2 there is an integer $N$ such that for all $i>N$, there is a diffeomorphism $f_{i}^{k}: B^{k}(r):=B_{N}^{k}(r) \rightarrow B_{i}^{k}(r)$ so that the pullback metric tensors $\left(f_{i}^{k}\right)^{*}\left(\exp _{p_{k}^{i}}^{*}\left(g_{i}\right)\right)$ converge in $L^{2, p_{-}}$class (cf. [Ya])

Let $\hat{\phi}_{i}=\left(f_{i}^{k}\right)^{*}\left(\exp _{p_{k}^{i}}^{*}\left(\phi_{i}\right)\right) \in \Gamma\left(\hat{S}^{+}\right)$be the pullback spinors, where $\hat{S}^{+}$is a spinor bundle of $B^{k}(r)$, which is isomorphic to the pullback spinor bundles $\hat{S}_{i}^{ \pm}$on $B^{k}(r)$ for all $i$ sufficiently large (passing to a subsequence if necessary.)

LEMMA 2.4. Let $M_{i}$ be as above. If $\lim _{i} \varepsilon_{i}=0$, then a subsequence of $\hat{\phi}_{i}$ above converges in $L^{2, p}$-class (for any integer $p>1$ ) to a non-trivial parallel harmonic spinor $\hat{\phi}$ with respect to the limit metric $g_{\infty}$.

Proof. Recall that all $\hat{\phi}_{i}$ satisfy

$$
\nabla_{i}^{*} \nabla_{i} \hat{\phi}_{i}+\frac{1}{4} s_{g_{i}} \hat{\phi}_{i}=0
$$

By Theorem 1.2 it follows that in the harmonic coordinate ball $B^{k}(r)$ and a local frame of sections of $\hat{S}^{+}$(resp. $\hat{S}^{-}$), (2.4.1) gives a second order uniformly elliptic equation, such that bounded;

(2.4.2) the coefficients of the second order term are uniformly $C^{1, \alpha_{-}}\left(\operatorname{resp} . L^{2, p_{-}}\right)$

(2.4.3) the coefficients of the first order terms (also the quadratic terms) are uniformly $C^{0, \alpha}$ - (resp. $L^{1, p_{-}}$) bounded;

(2.4.4) the coefficients of the zero order terms are uniformly $L^{p}$-bounded;

By $[\mathrm{ADN}]$ we know that $\left\|\hat{\phi}_{i}\right\|_{L^{2, p}} \leq C\left\|\hat{\phi}_{i}\right\|_{L^{\infty}}$, where $C$ is a universal constant. Therefore, by Lemma $2.3 \hat{\phi}_{i}$ contains a convergence subsequence in $L^{2, p^{\prime}}-\left(\right.$ resp. $\left.C^{1, \alpha}-\right)$ topology for any $p^{\prime}<p$, noting that there is a Sobolev embedding $L^{2, p} \subset C^{1,1-\frac{n}{p}}$.

Next we prove that the limit $\hat{\phi}$ is parallel with respect to the metric $g_{\infty}$. By $(2.2)$ $\hat{\phi}$ is not zero and the desired result follows.

Integrating (2.4.1) we get

$$
0=\frac{1}{\operatorname{vol} M_{i}} \int_{M_{i}}\left|\nabla_{i} \phi_{i}\right|^{2}+\frac{1}{4 \operatorname{vol} M_{i}} \int_{M_{i}} s_{g_{i}}\left|\phi_{i}\right|^{2}
$$

Since $\varepsilon_{i} \rightarrow 0$, the second term

$$
\lim _{i \rightarrow \infty} \inf \frac{1}{4 \operatorname{vol} M_{i}} \int_{M_{i}} s_{g_{i}}\left|\phi_{i}\right|^{2}=0
$$

Hence, passing to a subsequence we may assume that

$$
\lim _{i \rightarrow \infty} \frac{1}{\operatorname{vol} M_{i}} \int_{M_{i}}\left|\nabla_{i} \phi_{i}\right|^{2}=0
$$

We now use a trick in [Ya]. Suppose that $\hat{\phi}$ is not parallel with respect to $g_{\infty}$. Set $\hat{a}_{i}=\left|\hat{\nabla}_{i} \hat{\phi}_{i}\right|^{2}, \hat{a}=\left|\hat{\nabla}_{\infty} \hat{\phi}\right|^{2}$. Then there is a point $x \in B^{k}(r)$ for some $k$ such that $\hat{a}>b>0$ on the radius $\delta$ ball $B_{\delta}\left(x,\left(B^{k}(r), \hat{g}_{\infty}\right)\right)$ for some positive constants $b$ and $\delta$. Thus $\hat{a}_{i}>\frac{b}{2}$ on $B_{\delta}\left(x,\left(B^{k}(r), \hat{g}_{i}\right)\right)$ for $i$ large. Set $a_{i}=\left|\nabla_{i} \phi_{i}\right|^{2}$. By the Bishop-Gromov 
volume comparison theorem we get

$$
1-\frac{\operatorname{vol}\left\{a_{i} \leq \frac{b}{2}\right\}}{\operatorname{vol}\left(M_{i}\right)} \geq \frac{\operatorname{vol} B_{\delta}\left(q_{i}, M_{i}\right)}{\operatorname{vol}\left(M_{i}\right)} \geq \frac{\alpha(\delta)}{\alpha(D)}
$$

where $q_{i}=\exp _{p_{k}^{i}} \circ f_{i}^{k}(x)$ and $\alpha(r)$ is the volume function of radius $r$ ball in the hyperbolic space of curvature $-\lambda(n)$. Therefore

$$
\frac{\operatorname{vol}\left\{a_{i}<\frac{b}{2}\right\}}{\operatorname{vol}\left(M_{i}\right)} \leq 1-\frac{\alpha(\delta)}{\alpha(D)}
$$

for sufficiently large $i$. On the other hand, by $\left(^{*}\right)$ above, for any fixed $b>0$, it holds that

$$
\lim _{i \rightarrow \infty} \frac{\operatorname{vol}\left\{a_{i}<\frac{b}{2}\right\}}{\operatorname{vol}\left(M_{i}\right)}=1
$$

A contradiction.

3. Proof of Theorem A. Let $M$ be a Spin manifold, and $\phi \in \Gamma\left(S^{+}\right)$be a spinor. Using the first Bianchi identity we get

$$
\sum_{j} e_{j}\left(\nabla_{e_{i}} \nabla_{e_{j}}-\nabla_{e_{j}} \nabla_{e_{i}}-\nabla_{\left[e_{i}, e_{j}\right]}\right) \phi=-\frac{1}{4} \sum_{j, k, l} e_{j} R_{i j k l} e_{k} e_{l} \phi=-\frac{1}{2} \sum_{l} \operatorname{Ric}\left(e_{i}, e_{l}\right) e_{l} \phi
$$

for any $i$, where $e_{1}, \cdots, e_{n}$ is an orthonormal basis.

Proof of Theorem $A$. Suppose not. Then there is a sequence of Riemannian manifolds $\left(M_{i}, g_{i}\right)$ with $\mathcal{A}\left(M_{i}\right) \neq 0$ so that

$$
\operatorname{diam}\left(M_{i}\right) \leq 1, s_{g_{i}} \geq-i^{-1}, \operatorname{Sec}_{g_{i}} \leq 1
$$

but no one of $M_{i}$ admits the desired metrics.

By the Atiyah-Singer index theorem, the index $\operatorname{Ind}\left(D_{i}\right) \neq 0$. Therefore there is a nonzero harmonic spinor $\phi_{i} \in \operatorname{ker} D_{i}$, where $D_{i}$ is the Dirac operator. By normalizing we may assume (2.2) for all $\phi_{i}$.

Applying (3.1) to $\phi_{k}$ and integrating both sides we get

$$
\lim _{k \rightarrow \infty} \frac{1}{\operatorname{vol} M_{k}} \int_{M_{k}}\left|\sum_{l} \operatorname{Ric}\left(e_{i}, e_{l}\right) e_{l} \phi_{k}\right|^{p}=0
$$

by Lemmas 2.3 and 2.4 .

Since $\hat{\phi}$ is parallel with respect to $g_{\infty}$ and

$$
\frac{1}{\operatorname{vol} M_{k}} \int_{M_{k}}\left|\phi_{k}\right|^{4}=1
$$

the $C^{0}$-norm

$$
\left|\phi_{k}\right|_{C^{0}} \geq \frac{1}{2}
$$

for $k$ large. Therefore (3.2) implies that

$$
\lim _{k \rightarrow \infty} \frac{1}{\operatorname{vol} M_{k}} \int_{M_{k}}\left|\sum_{l} \operatorname{Ric}\left(e_{i}, e_{l}\right) e_{l}\right|^{p}=0
$$


for any $i$ and any prescribed integer $p>1$. This proves that the limit metric $g_{\infty}$ on $X$ is $L^{p}$-Ricci flat. By Theorem 1.3 this implies that $g_{\infty}$ is a smooth Ricci flat metric.

By Lemma 2.4 the limit of $\phi_{i}$ is a parallel spinor $\phi_{\infty}$ with respect to $g_{\infty}$. By [Fu] we know that the holonomy of $g_{\infty}$ must be one of the desired types.

\section{REFERENCES}

[ADN] S. AGMon; A. Douglis; L. NirenBerg, Estimates near the boundary for solutions of elliptic partial differential equations satisfying general boundary conditions II, Commun. on Pure. Appl. Math., 17 (1964), pp. 35-92.

[An] M. Anderson, Convergence and rigidity of manifolds under Ricci curvature bounds, Invent. Math., 102 (1990), pp. 429-445.

[CFG] J. Cheeger; K. Fưkaya; M. Gromov, Nilpotent structures and invariant metrics on collapsed manifolds, J.A.M.S, 5 (1992), pp. 327-372.

[CGr] J. CHEEGER; D. GROMOLL, The splitting theorem for manifolds of nonnegative Ricci curvature., Jour. Diff. Geom., 6 (1972), pp. 119-128.

[Fu] A. FutAKI, Scalar-flat closed manifolds not admitting positive scalar curvature metrics, Invent. Math., 112 (1993), pp. 23-29.

[Ga] S. GALlot, A Sobolev inequality and some geometric applications, Spectra of Riemannian manifolds, Kaigai, Tokyo (1983), pp. 45-55.

[GLP] M. Gromov, J. Lafontaine; P.PANSU, Structures metriques pour les varietes riemannienes, CedicFernand Paris (1981).

[JK] J. Jost; H. KARCHER, Geometrische methoden zur Gewinnug von a-priori- Schrander für harmonitsche Abbildungen, Manuscripta. Math., 40 (1982), pp. 27-77.

[Li] P. LI, On the Sobolev constant and the p-spectrum of a compact Riemannian manifold, Ann. Sci. École Norm. Sup., 13 (1980), pp. 451-468.

[Lic] P. Lichnerowicz, Théorie globale des connexions et des groupes d'holonomie, Dunod, Paris (1955).

[Lo] J. LohkAmp, Scalar curvature and hammocks, Math. Ann., 313 (1999), pp. 385-407.

[Lot] J. LotT, Â-genus and collapsing, J. Geom. Anal., 10 (2000), pp. 529-543.

[LM] B. Lawson and S. Michelson, Spin Geometry, Princeton Univ Press (1988).

[Pe] P. Petersen, Riemannian geometry, GTM, Springer-Verlag (1997).

[SY] R. SCHOEN; S. T. YAU, On the structure of manifolds with positive scalar curvature, Manuscripta Math., 28 (1979), pp. 159-183.

[St] S. Stolz, Simply connected manifolds of positive scalar curvature, Ann. of Math. (2), 136 (1992), pp. 511-540.

[Wa] M. Y. WANG, On non-simply connected manifolds with non-trivial parallel spinors, Ann. Global. Anal. Geom. (1995), pp. 31-42.

[Wi] E. WitTen, Monopoles and four-manifolds, Math. Res. Lett., 1 (1994), pp. 769-796.

[Ya] T. YamaguchI, Manifolds of almost nonnegative Ricci curvature, Jour. Diff. Geom., 28 (1988), pp. 157-167. 